\title{
Conhecimento de puérperas internadas em um alojamento conjunto acerca do aleitamento materno
}

\author{
Flávia Marinho Alves \\ Servidora pública naSecretaria Municipal de Saúde de Brejo da Madre de Deus \\ Graduanda em Enfermagem pelo Centro Universitário do Vale do Ipojuca - UNIFAVIP|DeVry \\ 凹lavia marinhobb@hotmail.com \\ Thaís Raiane Felix de Oliveira \\ Enfermeira pelo Centro Universitário do Vale do Ipojuca - UNIFAVIP|DeVry \\ \taty_rayanni@hotmail.com \\ Gésica Kelly da Silva Oliveira \\ Preceptora de estágios do Curso de Bacharelado em Enfermagem \\ do Centro Universitário do Vale do Ipojuca - UNIFAVIP|DeVry \\ Especialista em Urgência e Emergência pelo Centro \\ de Ensino, Formação, Aperfeiçoamento Profissional e Pesquisa - CEFAPP \\ Enfermeira pelo UNIFAVIP|DeVry \\ Mestranda em Educação em Ciências e Matemática pela UFPE - CAA \\ Pós-graduanda em Enfermagem (Ginecologia e Obstetrícia) \\ $\triangle$ gesicakelly.oliveira@gmail.com \\ Gleidson Monteiro dos Santos \\ Coordenador de estágios do Curso de Bacharelado em Enfermagem \\ do Centro Universitário do Vale do Ipojuca - UNIFAVIP|DeVry \\ Especialista em Urgência e Emergência, e em Terapia Intensiva, pelo Centro \\ de Ensino, Formação, Aperfeiçoamento Profissional e Pesquisa - CEFAPP \\ Enfermeiro pelo UNIFAVIP|DeVry \\ Mestrando em Educação em Ciências e Matemática pela UFPE - CAA \\ $\bowtie$ gleidsonmonteiro1@gmail.com
}

Recebido em 02 de fevereiro de 2017

Aceito em 13 de julho de 2017

\begin{abstract}
Resumo:
Esta pesquisa teve como objetivo analisar o conhecimento de puérperas internadas em uma maternidade a cerca do aleitamento materno. Trata-se de um estudo, quantitativo com abordagem descritiva, os dados foram obtidos a partir de questionário estruturado com perguntas objetivas, coletadas de fontes primárias. Este trabalho foi submetido e aprovado pelo Comitê de Ética em Pesquisa, através da Plataforma Brasil, em respeito a resolução nํ466/2016 do Conselho Nacional de Saúde. Os dados evidenciaram que as mães reconhecem os benefícios e as vantagens do aleitamento materno, o nível socioeconômico e a escolaridade parece não estar interligados aos conhecimentos sobre o leite materno e a vontade de amamentar. A pesquisa apontou ainda que o apoio familiar é um fator que colabora para a diminuição no abandono da amamentação. Assim, fica evidente a importância da educação em saúde constante durante o pré-natal, pós-parto e consultas de puericultura para garantir o sucesso desta relevante prática.
\end{abstract}

Palavras-chave: Aleitamento materno; Desmame; Enfermagem. 


\title{
Knowledge of inhered puerpers in a joint accommodation about maternal breastfeeding
}

\begin{abstract}
:
This research had as objective to analyze the knowledge of puerperas interned in a maternity center about breastfeeding. It is a quantitative study with a descriptive approach, the data were obtained from a structured questionnaire with objective questions, collected from primary sources. This study was submitted and approved by the Research Ethics Committee, through the Brazilian Platform, in compliance with Resolution 466/2016 of the National Health Council. Data showed that mothers recognize the benefits and advantages of breastfeeding, the level Socioeconomic and schooling appear to be unrelated to knowledge about breast milk and the willingness to breastfeed. The research also pointed out that family support is a factor that contributes to the decrease in the abandonment of breastfeeding. Thus, it is evident the importance of constant health education during prenatal, postpartum and childcare consultations to ensure the success of this relevant practice.
\end{abstract}

Keywords: Breastfeeding; Weaning; Nursing.

\section{Conocimiento de puérperas internadas en un alojamiento conjunto sobre la lactancia materna}

\section{Resumen:}

Esta investigación tuvo como objetivo analizar el conocimiento de puérperas internadas en una maternidad a cerca de la lactancia materna. Se trata de un estudio cuantitativo con enfoque descriptivo, los datos se obtuvieron a partir de un cuestionario estructurado con preguntas objetivas, recogidas de fuentes primarias. Este trabajo fue sometido y aprobado por el Comité de Ética en Investigación, a través de la Plataforma Brasil, en respeto a la resolución nº 466/2016 del Consejo Nacional de Salud. Los datos evidenciaron que las madres reconocen los beneficios y las ventajas de la lactancia materna, el nivel Socioeconómico y la escolaridad parecen no estar interconectados a los conocimientos sobre la leche materna y la voluntad de amamantar. La investigación apuntó además que el apoyo familiar es un factor que colabora para la disminución en el abandono de la lactancia. Así, queda evidente la importancia de la educación en salud constante durante el prenatal, posparto y consultas de puericultura para garantizar el éxito de esta relevante práctica.

Palabras clave: Lactancia materna; Desmame; Enfermería.

\section{INTRODUÇÃO}

Em torno do leite materno estudos científicos vêm, ao longo dos anos, comprovando sua superioridade aos demais leites e o seu poder de proteção contra infecções, dentre os inúmeros benefícios, as pesquisas apontam uma diminuição significativa na mortalidade infantil em crianças que receberam aleitamento materno exclusivo (AME) até os seis meses de vida (BRASIL, 2009).

Essas vantagens possibilitadas através do aleitamento não envolvem apenas a criança e vão além de uma criação de vínculo afetivo entre mãe/bebê, abrange fatores 
biológicos, históricos e sociais, criando ideologias e crenças a respeito deste hábito (FONSECA et al., 2011).

Na perspectiva de promover a efetivação desta prática foram criadas estratégias para o estímulo do aleitamento materno exclusivo, dentre elas a motivação advinha do conhecimento sobre a temática adquirida pela mulher é o principal fator que influência na decisão de amamentar, essas concepções são formadas ao longo da vida, iniciando na infância ao presenciar familiares amamentando, através de apoio marido/companheiro, seguido por saberes socioculturais e conhecimentos adquiridos durante a assistência de prénatal nas consultas e por meio de palestras oferecidas nas Unidades de Saúde da Família (TAKUSHI, 2008).

Embora, exista o incentivo de profissionais de saúde e programas destinados ao estímulo do aleitamento materno exclusivo até os seis meses de vida, a interrupção precoce continua a acontecer de forma significativa, pesquisas demonstram que a falta de conhecimento das mães ainda constitui o principal fator que contribui para o desmame precoce (ARANTES, 2011).

Todavia a falta do conhecimento não é a única causa do desmame precoce, outros fatores parecem estar relacionados, tais como a faixa etária da mãe, o nível de escolaridade, empregabilidade, além dos mitos comuns que questionam se o leite é fraco, se vai saciar a fome da criança, a pouca produção de leite, dentre outros (PERCEGONI, 2002).

Neste contexto, o apoio e a orientação destas puérperas e dos seus familiares, desde o pré-natal até o puerpério, requer comprometimento dos profissionais de enfermagem para garantir a efetivação da prática do aleitamento, sendo assim, é imprescindível o incentivo desta, ressaltando sua importância e seus benefícios, uma vez que se trata de uma estratégia inteligente e natural, além de econômica e eficaz para o desenvolvimento saudável da criança (MONTESCHIO; GAÍVA; MORREIRA, 2015).

Os profissionais de enfermagem que atuam nas Unidades de Saúde da família, devem realizar efetivamente, em sua práxis cotidiana, estratégias de educação em saúde, conscientização e aconselhamento no pré-natal de baixo risco, sendo esta uma das atribuições do enfermeiro estabelecido pelo Decreto no 94.406/87, que regulamenta a Lei do Exercício profissional da Enfermagem (BRASIL, 1987). 
Para Carrara e Oliveira (2013) é o enfermeiro que trabalha junto à sociedade e possui conhecimento técnico e científico para realizar estratégias de saúde que propiciem a efetivação de práticas de conscientização relacionadas a temática em questão, uma vez que estas atividades são fundamentais para a redução nas dificuldades, dúvidas e receios das nutrizes relacionados ao aleitamento materno, evitando assim a baixa adesão deste (AZEVEDO et al., 2015)

Nestas perspectivas, este estudo objetivou analisar o conhecimento das puérperas acerca do aleitamento materno, suas percepções em relação aos benefícios e dificuldades do aleitamento materno exclusivo nos primeiros seis meses de vida, bem como identificar se este público recebeu informações inerentes a esta atividade durante o acompanhamento pré-natal.

\section{METODOLOGIA}

Trata-se de um estudo epidemiológico transversal, quantitativo com abordagem descritiva. De acordo com Pereira (2006) os estudos transversais proporcionam objetividade na coleta dos dados, descrevendo características de um grupo em um determinado momento que podem ser analisadas em curto período de tempo. Para Gil (2009) a pesquisa descritiva visa conhecer e interpretar a realidade, por meio da descrição, classificação e estabelecimento de relações entre as variáveis e tem como característica a utilização de técnicas padronizadas para a coleta de dados como, por exemplo, o questionário. A pesquisa foi realizada na maternidade do Hospital Municipal Casa de Saúde Bom Jesus, situado na zona urbana do município de Caruaru-PE, este foi escolhido por ser referência no atendimento ao parto humanizado

$\mathrm{O}$ universo estudado contemplou as mulheres em período pós-parto, internadas no Alojamento Conjunto do Hospital Municipal Casa de Saúde Bom Jesus. Com base no quantitativo de partos realizados mensalmente nesta unidade (em média 350 partos) foi realizado o cálculo de amostra aleatória simples utilizando nível de confiança de 95\% e erro de estimação de $10 \%$, através do cálculo foi obtido a amostra necessária, sendo esta composta por 76 puérperas. 
Foram excluídos da pesquisa puérperas menores de 18 anos de idade, alocadas em outros setores do hospital, mulheres que estavam internadas por outras causas, não relacionadas ao parto e as participantes que se recusaram a assinar o Termo de Consentimento Livre e Esclarecido (TCLE). Para as mães não alfabetizadas foi realizado leitura de questionário, bem como assinalado as respostas de acordo com a opinião das participantes, nestes casos foi requerido a impressão digital dos participantes no TCLE.

Os dados foram coletados por meio de questionário estruturado com perguntas objetivas, no intuito de identificar a faixa etária, escolaridade, renda, estado civil, números de filhos, a participação em atividades de educação em saúde durante o pré-natal e a percepção das puérperas em relação aos benefícios do aleitamento materno exclusivo nos primeiros seis meses de vida.

Após a coleta dos dados os mesmos foram duplamente digitados no Programa Microsoft Excel ${ }^{\circledR}$ versão 2010, em busca de erros e/ou omissões. A análise dos dados aconteceu por meio da avaliação das respostas objetivas. Os dados quantificáveis foram analisados descritivamente para obtenção de frequências absolutas e relativas, estes foram apresentados de forma tabular, por meio do Programa Microsoft Excel ${ }^{\oplus}$ versão 2010.

Este trabalho foi autorizado pela Secretaria Municipal de Saúde e pelo Comitê de Ética em Pesquisa, através da Plataforma Brasil, sob Certificado de Apresentação para Apreciação Ética (CAAE), nº 56954416.3.0000.5666. Após aprovação dos órgãos supracitados a pesquisadora visitou o Hospital Municipal Casa de Saúde Bom Jesus, onde foi solicitada a resposta do questionário as puérperas internadas no alojamento conjunto. Antes da coleta de dados os participantes da pesquisa foram informados sobre os objetivos desta pesquisa, riscos e benefícios que está trará a sociedade, além de serem instruídos quanto à assinatura do Termo de Consentimento Livre e Esclarecido.

\section{RESULTADOS}

A maioria das participantes encontrava-se na faixa etária de 18 à 24 anos representando 55\% (41), 25 à 30 anos 30\% (23), 31 à 35 anos 8\% (6), 36 à 40 anos 7\% (5). Quanto ao o estado civil das puérperas, a união estável mostrou-se predominante com 52\% (39), seguida pelas casadas $25 \%$ (19), solteiras $21 \%$ (16), divorciadas $1 \%$ (1) e viúvas $1 \%$ (1). 
No que concerne ao tempo de estudo observou-se que a maioria das puérperas possuía ensino fundamental incompleto $40 \%$ (30), seguida pelas que possuem o ensino fundamental completo $33 \%$ (25), ensino médio completo $21 \%$ (16), ensino superior completo $4 \%$ (3), não estudou $1 \%$ (1), apenas alfabetizada $1 \%$ (1).

Da amostra pesquisada 78\% (59) das mães residem com companheiros e filhos, $16 \%$ moram com familiares $16 \%$ (12) e $6 \%$ (5) somente com os filhos.

Em relação à profissão 59\% (45) das mulheres pesquisadas afirmam trabalhar fora do lar e $41 \%$ (31) relataram que não exercem atividades laborais. Dentre as que trabalham as principais profissões encontradas foram: costureira 31\% (14), agricultora $16 \%(7)$, atendente 9\% (4), outras profissões $44 \%$.

Considerando a renda familiar, foi evidenciado que a maioria sobrevive com até 1 salário mínimo $72 \%$ (55), seguida de até 2 salário mínimo 25\% (19) e até 3 salários mínimos $3 \%(2)$.

A tabela 1 representa os dados acima mencionados, contemplando idade materna, estado civil escolaridade, composição familiar, profissão, tipos de profissão e renda familiar.

Quando questionadas sobre a participação das puérperas nas atividades de educação em saúde sobre aleitamento materno 79\% (60) relataram que receberam orientação por um profissional de saúde sobre Aleitamento Materno durante a gestação, 21\% (16) não receberam nenhuma orientação.

Sobre o conhecimento geral sobre o aleitamento materno das puérperas evidenciouse que 93\% (71) acreditam que o leite materno possui todos os nutrientes necessários a saúde do bebê, $4 \%$ (3) que ele não é suficiente para acabar com a fome do bebê, 3\% (2) que é mais fraco que o leite de vaca.

Quando interrogadas sobre os motivos que levariam ao abandono da amamentação $11 \%$ (8) relataram que deixaria de amamentar se o bico do peito rachasse, $1 \%$ (1) se considerasse que o leite está fraco, 5\% (4) se tivesse com pouco leite e 83\% (63) relataram que não deixaria de amamentar por nenhum dos motivos citados acima. 
Tabela 1 - Distribuição das características socioeconômica e demográfica das puérperas participantes da pesquisa. Caruaru, PE, Brasil, 2016

\begin{tabular}{|c|c|c|}
\hline IDADE MATERNA & (n) & $\%$ \\
\hline 18 à 24 & 41 & $55 \%$ \\
\hline 25 à 30 & 23 & $30 \%$ \\
\hline 31 à 35 & 6 & $8 \%$ \\
\hline 36 à 40 & 5 & $7 \%$ \\
\hline Total & 76 & $100 \%$ \\
\hline \multicolumn{3}{|l|}{ ESTADO CIVIL } \\
\hline Solteira & 16 & $21 \%$ \\
\hline Casada & 19 & $25 \%$ \\
\hline Divorciada & 1 & $1 \%$ \\
\hline Viúva & 1 & $1 \%$ \\
\hline União Estável & 39 & $52 \%$ \\
\hline Total & 706 & $100,00 \%$ \\
\hline \multicolumn{3}{|l|}{ ESCOLARIDADE } \\
\hline Não estudei & 1 & $1 \%$ \\
\hline Alfabetizada & 1 & $1 \%$ \\
\hline Fundamental Incompleto & 30 & $40 \%$ \\
\hline Fundamental Completo & 25 & $33 \%$ \\
\hline Ensino Médio Completo & 16 & $21 \%$ \\
\hline Superior Completo & 3 & $4 \%$ \\
\hline Total & 76 & $100 \%$ \\
\hline \multicolumn{3}{|c|}{ PESSOAS RESIDENTES NO MESMO DOMICÍLIO } \\
\hline Companheiro e filhos & 59 & $78 \%$ \\
\hline Familiares & 12 & $16 \%$ \\
\hline Filhos & 5 & $6 \%$ \\
\hline Total & 76 & $100,00 \%$ \\
\hline \multicolumn{3}{|l|}{ PROFISSÃO } \\
\hline Sim & 45 & $59 \%$ \\
\hline Não & 31 & $41 \%$ \\
\hline Total & 76 & $100 \%$ \\
\hline
\end{tabular}




\begin{tabular}{ccc}
\hline TIPOS DE PROFISSÃo & (n) & \% \\
\hline Costureira & 14 & $31 \%$ \\
Agricultora & 7 & $16 \%$ \\
Atendente & 4 & $9 \%$ \\
Outras & 20 & $44,00 \%$ \\
\hline RENDA FAMILIAR & (n) & $\%$ \\
\hline Até 1 Salário Mínimo & 55 & $72 \%$ \\
Até 2 Salários Mínimos & 19 & $25 \%$ \\
Até 3 Salários Mínimos & 2 & $3 \%$ \\
Total & 76 & $100 \%$ \\
\hline
\end{tabular}

Fonte: Os Autores, 2016.

No que se referente aos benefícios e malefícios da chupeta 51\% (39) não deve ser utilizada, pois prejudica o desenvolvimento motor oral da criança, 22\% (17) deve ser utilizada, pois acalma o bebê e não traz prejuízo algum para o mesmo e 27\% (20) não souberam informar.

No caso de retornar ao trabalho ou na necessidade de se ausentar da criança 60\% (46) das mulheres investigadas relataram que deixariam o leite humano armazenado na geladeira ou congelador e $40 \%$ (30) ofereceriam a mamadeira com outro tipo de leite.

A tabela 2 apresenta os dados supracitados, relacionados à participação das puérperas em atividades de educação em saúde mostrando se as mesmas receberam orientações sobre aleitamento materno, à percepção das pesquisadas sobre o leite materno, os motivos para abandono da amamentação, os dados sobre o uso da chupeta e a alimentação na ausência da mãe.

Tabela 2 - Distribuição segundo à participação das puérperas em educação em saúde, dos conhecimentos em relação ao aleitamento materno e o uso da chupeta. Caruaru, PE, Brasil, 2016

\begin{tabular}{cccc}
\hline PARTICIPAÇÃO EM ATIVIDADE DE EDUCAÇÃO EM SAÚDE & (n) & $\%$ \\
\hline Sim & 60 & $79 \%$ \\
Não & 16 & $21 \%$ \\
Total & 76 & $100 \%$ \\
& & & \\
\hline
\end{tabular}




\begin{tabular}{|c|c|c|}
\hline PERCEPÇÃO SOBRE O LEITE MATERNO & $(\mathrm{n})$ & $\%$ \\
\hline Possui todos os nutrientes & 71 & $93 \%$ \\
\hline Não é suficiente & 3 & $4 \%$ \\
\hline É mais fraco que o leite de vaca & 2 & $3 \%$ \\
\hline Total & 76 & $100 \%$ \\
\hline \multicolumn{3}{|l|}{ ABANDONO DA AMAMENTAÇÃO } \\
\hline O bico do peito rachou & 8 & $11 \%$ \\
\hline Considera o leite fraco & 1 & $1 \%$ \\
\hline Teve pouco leite & 4 & $5 \%$ \\
\hline Não deixaria de amamentar & 63 & $83 \%$ \\
\hline Total & 76 & $100 \%$ \\
\hline \multicolumn{3}{|l|}{ USO DA CHUPETA } \\
\hline $\begin{array}{c}\text { Deve ser utilizada, pois acalma o bebê e não traz prejuízo algum para o } \\
\text { mesmo }\end{array}$ & 17 & $22 \%$ \\
\hline $\begin{array}{l}\text { Não deve ser utilizada, pois prejudicam o desenvolvimento motor oral da } \\
\text { criança }\end{array}$ & 39 & $51 \%$ \\
\hline Não souberam informar & 20 & $27 \%$ \\
\hline Total & 76 & $100 \%$ \\
\hline \multicolumn{3}{|l|}{$\begin{array}{c}\text { NO CASO DO RETORNO AO TRABALHO OU NECESSIDADE DE SE } \\
\text { AUSENTAR DA CRIANÇA }\end{array}$} \\
\hline Deixaria o leite armazenado na geladeira ou congelador & 46 & $60 \%$ \\
\hline Ofereceria a mamadeira com outro tipo de leite & 30 & $40 \%$ \\
\hline Total & 76 & $100 \%$ \\
\hline
\end{tabular}

Fonte: Os Autores, 2016.

\section{DISCUSSÃo}

Este trabalho avaliou uma amostra das puérperas atendidas em uma maternidade de um Hospital de referência em parto humanizado. 
$\mathrm{Na}$ amostra estudada a faixa etária de maior prevalência compreendeu as nutrizes de 18 à 24 anos correspondendo a 55\% dos pesquisados. Estudos mostram que a idade materna pode influenciar na amamentação, mas que não indica um fator de risco para a ausência de conhecimento materno sobre o aleitamento, visto que as mães adolescentes tendem a ter menos disposição para amamentar, porém são mais sensíveis ao ato de amamentar que as mulheres com mais idade, necessitando de atendimento e acolhimento mais atento e efetivo (MOURA, 2015).

Entretanto, ainda segundo Moura (2015), existe outros fatores que podem estar relacionado ao desmame precoce, tais como: ausência de orientação prévia sobre amamentação, nível de escolaridade, tendo uma menor compreensão da importância do aleitamento materno exclusivo ou influência dos conhecimentos empíricos.

A situação conjugal das entrevistadas apontou que 52\% encontram-se em união estável e 25\% são casadas. Para Simões et al., (2015) o convívio e a presença do companheiro torna-se importante para o fortalecimento do vínculo familiar, visto que mulheres que não possuem companheiros estão mais predispostas a amamentarem seus filhos por um período menor de tempo.

O fato das mulheres desfrutarem de apoio familiar, sendo do pai do bebê ou de outro membro da família, consta como fator que coopera para o baixo risco do abandono precoce da amamentação uma vez que, esse apoio atua como fator de estímulo, sendo importante para o incentivo e acolhimento durante todo o processo da lactação (TEIXEIRA; NITSCHKE; SILVA 2011).

Em relação à escolaridade materna o estudo revelou que 40\% das entrevistadas possuem o ensino fundamental incompleto e $33 \%$ ensino fundamental completo. Segundo Lacerda e Santos (2013) a escolaridade estabelece um fator que influência diretamente a motivação para amamentação exclusiva, visto que mulheres com um nível mais elevado de instrução amamentam seus filhos por mais tempo, o que parece estar relacionado ao discernimento das orientações em relação à importância do aleitamento materno exclusivo e os benefícios, tanto para a mãe quanto para a criança. Todavia, o presente estudo detectou que apesar da maioria das mães não possuírem ensino médio completo, estas detinham conhecimentos relevantes sobre a importância da lactação. 
Das puérperas pesquisadas 51\% estão inseridas no mercado de trabalho, para Araújo et al., (2008) a participação da mulher no mercado de trabalho permanece cada vez mais acentuada e não apresenta qualquer propensão para o declínio dessa inserção, o que acarreta cada vez mais nas mudanças dos hábitos sociais, tornando muitas vezes seu cotidiano mais ansioso e tenso, sendo esses fatores coadjuvantes para o aumento do desmame antes dos seis primeiros meses de vida.

Com relação à renda, este estudo evidenciou que a maioria das nutrizes recebem até um salário mínimo. Moura et al., (2015) afirma que o nível socioeconômico está diretamente associado com a amamentação, visto que as mulheres que apresentam uma situação econômica mais elevada inclinam-se a amamentar por períodos mais amplos.

Ao analisar a participação das mulheres em atividades de educação em saúde constatou-se que o índice de participação foi de $79 \%$ sendo esse valor considerado satisfatório. A educação em saúde durante o acompanhamento pré-natal e puerpério é uma estratégia imprescindível para o empoderamento das mulheres sobre a lactação, o Enfermeiro é um profissional que exerce influência relevante na efetivação desta prática, uma vez que acompanha as mulheres durante a gestação e promove atividades de educação em saúde na sua práxis, quer seja de maneira individual ou coletiva (CASTRO; SILVA; SILVA, 2015).

No que concerne a percepção do leite materno 93\% das puérperas demostraram saber que o leite materno possui todos nutrientes para o bebê, evidenciando conhecimento relevante sobre a temática abordada. Conforme Martins e Santana (2013) esse conhecimento não determina que o aleitamento materno seja realizado com êxito apontando a importância da continuação dos programas de promoção ao AME, juntamente com a assistência integral e efetiva a saúde da mulher que abrange o ciclo gravídicopuerperal, além de um cuidado global a saúde da criança.

Sobre os motivos que levam as mulheres a abandonar a lactação, $83 \%$ relataram que não deixaria de amamentar se houvesse fissuras no mamilo ou se considerasse o leite fraco ou insuficiente. Moraes et al., (2014) evidencia que a dor mamária, causada na maioria das vezes pelo ingurgitamento mamário e o choro excessivo do bebê são os grandes norteadores desse fato, revelando que durante as atividades de educação em saúde, além de enfatizar a importância dos benefícios do leite materno no crescimento e desenvolvimento da criança a 
abordagem em torno da forma correta da pega do bebê ao seio materno, as possíveis dificuldades e a duração da mamada se faz necessária.

No que se refere ao uso da chupeta $51 \%$ das nutrizes responderam que a mesma não deve ser utilizada, demostrando que uma percentual considerável das mulheres entrevistadas (49\%) ainda não possuíam saberes necessários sobre o uso da chupeta. Cardoso (2015) descreve que o uso da chupeta pode ocasionar deformidade da arcada dentária, prejudicando o desenvolvimento motor oral da criança, dificultando a sucção e favorecendo a diminuição das mamadas, além disso, pode promover a diminuição do estímulo nas terminações nervosas encontradas na aréola.

Quando questionadas em relação ao tipo de leite que seria ofertado a criança em caso de retorno ao trabalho ou necessidade da mãe se ausentar $40 \%$ responderam que ofereceria a mamadeira com outro tipo de leite. Diante disso, fica evidente a necessidade de maiores orientações voltadas também para o armazenamento bem como, a forma correta de ofertar o leite materno possibilitando que não exista a interrupção da amamentação quando a mulher retornar ao trabalho ou tenha a necessidade de se ausentar (ROCCI; FERNANDE, 2014).

\section{CONSIDERAÇÕES FINAIS}

Diante dos resultados obtidos permitiu-se explorar os conhecimentos das puérperas quanto a prática da amamentação e seus benefícios para a mãe e para o bebê, uma vez que o mesmo é um fator essencial para o desenvolvimento saudável do lactente.

Dentre os diversos fatores que estão relacionados a construção do conhecimento sobre a amamentação, à faixa etária das mulheres pesquisadas mostrou que a idade não influenciou na decisão de amamentar, verificou-se que o nível de escolaridade, bem como a situação econômica não demostraram serem fatores decisivos no conhecimento das mulheres sobre $o$ aleitamento.

Foi observado, ainda que as nutrizes possuem uma percepção satisfatória em torno dos benefícios da lactação e suas vantagens, todavia ainda existe a falta de empoderamento 
sobre a continuidade da oferta de leite materno na ausência da nutriz e os malefícios ocasionados pela utilização da chupeta.

Assim, é preciso reconhecer a importância da educação em saúde constante e eficiente com as mulheres durante o período pré-natal, puerperal e de puericultura, na perspectiva de garantir o sucesso da amamentação, portanto, é substancial a mobilização de toda equipe de saúde na pessoa do enfermeiro, uma vez que este é responsável por parte da equipe (Técnicos de Enfermagem e Agentes Comunitários de Saúde), para o fortalecimento de estratégias que condicionem cada vez mais a efetivação do AME, tendo em vista os benefícios relevantes que esta prática promove para a criança, família e comunidade.

\section{REFERÊNCIAS}

ARANTES, C. I. S. et.al. Aleitamento materno e práticas alimentares de crianças menores de seis meses em Alfenas. Revista de Nutrição, Minas Gerais, v.24 n.3, Mai./Jul., 2011. Disponível em: <http://www.scielo.br/pdf/rn/v24n3/a05v24n3.pdf>. Acessado em: 07 de Abril de 2016.

ARAÚJO, O. D. et. al. Aleitamento materno: fatores que levam ao desmame precoce. Revista brasileira de enfermagem, São Paulo, v. 61, n. 4, Jul./Ago. 2008 Disponível em: < http://bases.bireme.br/cgibin/wxislind.exe/iah/online/?

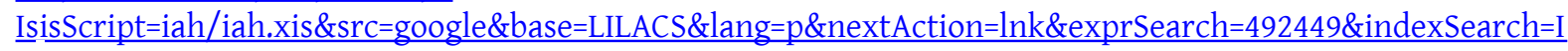

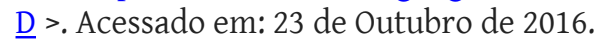

AZEVEDO, A. R. R. et.al. O manejo clínico da amamentação: saberes dos enfermeiros. Escola Anna Nery Revista de enfermagem, Rio de Janeiro, v.19, n.3 Jul/Set, 2015 Disponível em:< http://www.revistaenfermagem.eean.edu.br/detalhe artigo.asp?id=1319>. Acessado em: 4 de Abril de 2016.

BRASIL. Ministério da Saúde. Saúde da criança: nutrição infantil aleitamento materno e alimentação complementar. Cadernos de Atenção Básica, n. 23, Brasília, 2009.

BRASIL. Decreto n. ${ }^{\circ}$ 4.406, de 8 de junho de 1987, Regulamenta a lei do exercício da enfermagem, Disponível em :<http://www.planalto.gov.br/ccivil_03/decreto/1980-1989/D94406.htm>. Acessado em : 11 de Maio de 2016.

CARDOSO, A. M. Importância do aleitamento materno na perspectiva da grávida. Vila Real: UTAD. 2015. 60p. dissertação (mestrado em educação de saúde comunitária) - mestrado de enfermagem comunitária. Universidade de Trás-os-Montes e alto douro 2013. Disponível em: <https://repositorio.utad.pt/bitstream/10348/4672/1/msc_amcardoso.pdf>. Acesso em : 10 de Abril de 2016.

CASTRO, R. J. S.; SILVA, E. M. B.; SILVA, D. M. Percepção das mães sobre as práticas dos enfermeiros na promoção do aleitamento materno. Revista de Enfermagem Referência, Coimbra, v. 4, n. 6, Jul./Set. 2015. Disponível em: <http://dx.doi.org/10.12707/RIV14077>. Acessado em: 29 de Outubro de 2016.

FONSECA, M. O. et.al. Aleitamento materno: conhecimento de mães admitidas no alojamento conjunto de um hospital universitário. Ciência, cuidado e saúde, Triângulo Mineiro, v.10, n.1, Jan./Mar. 2011 Disponível em: 
<http://periodicos.uem.br/ojs/index.php/CiencCuidSaude/article/viewFile/11009/pdf>. Acessado em: 04 de Abril de 2016.

GIL, A. C. Como elaborar projetos de pesquisa. São Paulo: Atlas, 2009.

LACERDA, C. N.; SANTOS, S. M. J. Aleitamento materno exclusivo: O conhecimento das mães. Revista Brasileira de Educação e Saúde, Pombal, v. 3, n. 2, Abr./jun. 2013. Disponível em: $<$ https://scholar.google.com.br/scholar?q=Aleitamento+materno+exclusivo\%3A+O+conhecimento + das $+m$ \%C3\%A3es\&btnG $=\& h l=p t-B R \& a s \quad s d t=0 \% 2 C 5>$. Acessado em: 26 de outubro de 2016 .

MARTINS, M. Z:; SANTANA, L. S. Benefícios da amamentação para saúde materna. Interfaces Científicas-Saúde e Ambiente, Aracaju, v. 1, n. 3, Julho 2013. Disponível em:< https://scholar.google.com.br/scholar?q=BENEF $\%$ C3\%8DCIOS+DA+AMAMENTA\%C3\%87\%C3\%830+PARA+SA

\% $3 \% 9 \mathrm{ADE}+\mathrm{MATERNA}+$ Maria+Zilda+Oliveira+Martins1+Licia+Santos+Santana2\&btnG=\&hl=ptBR\&as sdt=0\%2C5>. Acessado em: 29 de Outubro de 2016.

PERCEGONI, N. et.al. Conhecimento sobre aleitamento materno de puérperas atendidas em dois hospitais de Viçosa, Minas Gerais. Revista de nutrição, Campinas, v.15 n.1, Jan./Abr. 2002 Disponível em: <http://www.scielo.br/pdf/rn/v15n1/a04v15n1.pdf>. Acessado em : 04 de Abril de 2016.

MOSTESCHIO, C. A. C.; GAIIVA, M. A. M.; MORREIRA, M. D. D. S. O. enfermeiro frente ao desmame precoce na consulta da enfermagem á criança. Revista Brasileira de Enfermagem, Mato Grosso, v.68, n.5, Set./Out. 2015 Disponível em: $<$ http://www.scielo.br/scielo.php?pid=S003471672015000500869\&script=sci_abstract\&tlng=es"tlng=es>. Acessado em: 04 de Abril de 2016.

MOURA, E. R. B. B. et.al. Investigação dos fatores sociais que interferem na duração do aleitamento materno exclusivo. Revista de Toxicologia, Risco Ambiental e Sociedade, São Paulo v. 8, n. 2, junho 2015 Disponível em: <http://revistarevinter.com.br/index.php/toxicologia/article/viewFile/203/418>. Acessado em: 04 de Novembro de 2016.

MORAES, J. T. et al. A percepção da nutriz frente aos fatores que levam ao desmame precoce em uma unidade básica de saúde de Divinópolis/MG. Revista de Enfermagem do Centro-Oeste Mineiro, Minas Gerais, v. 4, n. 1, Jan./Abr. 2014. Disponível em: <http://seer.ufsj.edu.br/index.php/recom/article/viewArticle/446>.

Acessado em: 03 de Novembro de 2016

PEREIRA, J. M. Manual de Metodologia da Pesquisa Científica. São Paulo: Atlas, 2006.

ROCCI, E.; FERNANDES, R. A. Q. Dificuldades no aleitamento materno e influência no desmame precoce. Revista Brasileira de Enfermagem, São Paulo, v.67, n.1, Jan./Fev. 2014 Disponível em: <http://www.scielo.br/pdf/reben/v67n1/0034-7167-reben-67-01-0022.pdf>. Acessado em: 09 de Abril de 2016.

SIMÕES, I. A. R. et al. Influência dos mitos e das crenças nas nutrizes quanto amamentação em uma Cidade do Vale do Paraíba. Revista Ciências sem Saúde, Minas Gerais, v. 5, n.3, Jul./Set., 2015 Disponível em: <http://200.216.240.50:8484/rcsfmit/ojs-2.3.3-3/index.php/rcsfmit_zero/article/view/385> . Acessado em: 29 de Outubro de 2016.

TAKUSHI, S. A. M. et.al. Motivação de gestantes para o aleitamento materno. Revista de nutrição, Campinas, v.21, n.5, Set./Out., 2008 Disponível em: <http://www.scielo.br/pdf/rn/v21n5/a02v21n5.pdf>. Acessado em: 04 de Abril 2016.

TEIXEIRA, M. A.; NITSCHKE, R. G.; SILVA, L. W. S. A prática da amamentação no cotidiano familiar-um contexto intergeracional: influência das mulheres-avós. Kairós Gerontologia. Revista da Faculdade de Ciências Humanas e Saúde, São Paulo, v. 14, n. 3, Junho, 2011 Disponível em: <

http://revistas.pucsp.br/index.php/kairos/article/view/6501/4713>. Acessado em: 29 de Outubro de 2016. 